\title{
New therapeutic approach by sirolimus for enteropathy treatment in patients with LRBA deficiency
}

\author{
${ }^{1}$ Department of Laboratory Medicine, Imam Hassan Mojtaba Hospital, Alborz University of Medical Sciences, Karaj, Iran \\ ${ }^{2}$ Research Center for Immunodeficiencies, Children's Medical Center, Tehran University of Medical Sciences, Tehran, Iran \\ ${ }^{3}$ Primary Immunodeficiency Diseases Network (PIDNet), Universal Scientific Education and Research Network (USERN), \\ Tehran, Iran \\ ${ }^{4}$ Division of Clinical Immunology, Department of Laboratory Medicine, Karolinska Institute at Karolinska University Ho- \\ spital Huddinge, Stockholm, Sweden \\ ${ }^{5}$ Department of Immunology, School of Medicine, Isfahan University of Medical Sciences, Isfahan, Iran \\ ${ }^{6}$ School of Advanced Technologies in Medicine, Department of Medical Biotechnology, Tehran University of Medical \\ Sciences, Tehran, Iran \\ ${ }^{7}$ Department of Biomedical Engineering, Faculty of New Sciences and Technologies, University of Tehran, Tehran, Iran
}

\section{KEY WORDS}

Sirolimus; enteropathy; LRBA deficiency; common variable immune deficiency; autoimmunity

\section{Corresponding author}

Asghar Aghamohammadi

Research Center for Immunodeficiencies,

Children's Medical Center, Tehran

University of Medical Sciences, Tehran, Iran

E-mail: aghamohammadi@sina.tums.ac.ir

Doi

10.23822/EurAnnACI.1764-1489.22

\section{Summary}

Purpose. To report the successful use of sirolimus for management of enteropathy in four patients with LPS-responsive beige-like anchor protein (LRBA) deficiency. Methods. Case series. Results. sirolimus therapy led to a complete improvement of symptoms including decrease in frequency and severity of diarrhea, as well as patients' weight gain. No signs of abdominal cramps and anorexia were also detected during the follow up period after treatment. Conclusions. sirolimus with its potential efficacy and immunomodulatory properties may be recommended for the treatment of severe enteropathy refractory to conventional therapy in patients with LRBA deficiency.

\section{Introduction}

LPS-responsive beige-like anchor protein (LRBA) deficiency is a rare genetic disorder caused by biallelic loss-of-function mutations in the $L R B A$ gene. This disorder is characterized by early-onset hypogammaglobulinemia, chronic diarrhea and autoimmune manifestations (1-4). Similar to common variable immune deficiency (CVID) patients, affected individuals show a reduced levels of immunoglobulin (Ig) isotypes and suffer from recurrent infections, hepatosplenomegaly, chronic pulmonary disorders as well as auto-inflammatory conditions including idiopathic thrombocytopenic purpura (ITP), autoimmune hemolytic anemia (AIHA) and enteropathy (1,5-9).

The enteropathy phenotype includes autoimmune enteropathy, inflammatory bowel disease (IBD)/IBD-like disease and non-infectious recurrent diarrhea. LRBA deficiency has been reported to be common among patients with CVID-like phenotype underwent genetic diagnosis $(2,10,11)$. CVID patients and patients with LRBA deficiency resemble symptoms of enteropathy presenting in immunocompetent individuals, but the pathology is usually documented to be not similar and the symptoms often do not respond to the conventional therapies. In LRBA deficient 
patients, chronic diarrhea is characterized by duodenal villous atrophy and large bowel lymphocytic infiltration (5). Recent studies have reported that the chronic and severe diarrhea in patients with LRBA deficiency may not improve despite intravenous Ig (IVIg) treatment $(6,12)$. Medical therapy typically with corticosteroids (budesonide and prednisone), empiric antibiotic therapy and gluten free diets have been used commonly (13). In patients refractory to corticosteroids, treatment with immunosuppressive drugs such as azathioprine, 6-mercaptopurine, tacrolimus, mycophenolate mofetil, infliximab, and rituximab have been reported. Side effects are commonly documented in administration of this group of medications, and maintaining remission has been reported to be unsuccessful in previous studies $(5,14-16)$. sirolimus, also known as rapamycin, is a macrocyclic lactone antibiotic which also has a profound immunosuppressive property on the cellular immune response, particularly on $\mathrm{T}$ cells. sirolimus binds to the same intracellular receptor as tacrolimus and cyclosporine, however does not inhibit calcineurin. sirolimus blocks the "mammalian target of rapamycin" (mTOR) which subsequently interrupts signaling pathways for several cytokines and growth factors including interleukin 2 (IL2). Recent studies have suggested the effectiveness of sirolimus to reduce chronic diarrhea in patients with entropathy. Here, we report for the first time the successful use of sirolimus for management of entropathy in four patients with LRBA deficiency.

\section{Case presentation}

Case 1. A 14 years old female patient with LRBA deficiency was diagnosed at the age of five years old with hypogammaglobulinemia. She is a child of related (first cousin) parents. Her first manifestation was diarrhea which started at six months of age. The patient underwent antibiotic therapy for the diarrhea but there was no improvement in her symptoms. Other manifestations included splenomegaly, hepatomegaly and juvenile rheumatoid arthritis at the age of four. She underwent treatment for immunodeficiency at five years of age with IVIg, accordingly her diarrhea was controlled. Diarrhea became more severe since a year ago, up to 20 times during the day and 8 times during the night, and consequently six kilograms-weight loss was detected. Infliximab was administrated for five months, but no improvement was observed in diarrhea and weight loss symptoms. The pathological report of the colonoscopy showed edema and excess infiltration of lamina propria with lymphocytes and eosinophils. Focal micro-abscess formation and cryptitis were also detected. Her microscopic reports were conclusive of mild chronic gastritis, esophagitis and active colitis but no parasite or Helicobacter pylori infection was reported.

Case 2. A 21-year-old LRBA deficient female patient with refractory diarrhea is a child from the first cousin related parents. The first presentation of her disease was diarrhea which started at the age of 13. She was diagnosed with celiac based on the endoscopic evaluation, thus she underwent gluten free diet, but there was no improvement in her symptoms. The patient has a history of other autoimmune complications including autoimmune thyroiditis, ITP and AIHA. IVIg therapy had been started two years ago which was not effective for her autoimmune symptoms. Splenectomy was performed one year ago for the treatment of the cytopenia. She was also on prednisolone treatment in the last year, but no improvement in her diarrhea and weight loss symptoms were seen. The patient experienced a nine kilograms weight loss and cachexia during the last year. The results of the endoscopic and colonoscopy samples were suggestive of celiac like enteropathy, chronic active gastritis and atrophic duodenal mucosa with villus atrophy.

Case 3. This case is a six years old female patient with LRBA deficiency and a history of recurrent diarrhea has been documented for this patient. She is a child from a first cousin related parents, with no family history of immunodeficiency. Until the age of four, the patient had no symptoms or signs of diarrhea. She was hospitalized at the ages of four and six years old, both due to chronic diarrhea and consequent severe dehydration. Frequency of diarrhea was more than 10 times a day (with an increased volume and wateriness). The pathologic report of the colonoscopy revealed cryptitis and crypt abscess associated with lymphocytic infiltration and neutrophils in the lamina propria. Results were suggestive of active colitis and especially of IBD.

Case 4. The forth case was a 27 years old male, born to consanguineous parents. His symptoms started at the age of two with respiratory tract infection (RTI) and identified with tentative diagnosis of CVID at the age of 10. He was under observation since age two, and several episodes of pneumonia, sinusitis and diarrhea were detected. Other complications included arthritis, bronchiectasis, failure to thrive and clubbing. Patient's diarrhea did not respond well to treatment with corticosteroids. Histopathologic evaluation showed infiltrations of lymphocytes, plasma cells and eosinophil cells in the lamina propria of the intestinal epithelium; suggestive of acute ileitis. Furthermore, infiltration of lymphocytes, plasma cells and polymorphonuclear leukocytes (PMNs) were reported in the colonic mucosa with preserved cryptic architecture which was suggestive of noncrypt destructive colitis.

Immunologic characteristics of four patients with LRBA deficiency are illustrated in table 1. For all patients, colonoscopy was performed and the diagnosis of enteropathy and acute colitis were confirmed. As prior therapy including infliximab, cotrimoxazole, clarithromycin and gluten free diet had failed to control the disease process, sirolimus therapy with the dosage of $1 \mathrm{mg} /$ day for three months was started for all patients. This treatment led to a complete improvement of their symptoms including decrease in frequency and severity of diarrhea and im- 
provement in the patients' weight. Evidence of a response trend is further documented by normal serum level of albumin, calcium and potassium.

\section{Discussion}

LRBA deficiency is characterized by combined immunodeficiency, enteropathy, and autoimmune complications. According to previously published studies, clinical features in patients with LRBA deficiency are heterogeneous, and first presentations of the disease often occur during childhood (2). The cohort study of Alkhairy et al. (2) divided the disease phenotypes into categories of RTI, autoimmunity, organomegaly, and enteropathy. They described the enteropathy phenotype as an overlapped group of autoimmune enteropathy, IBD/IBD-like disease and non-infectious diarrhea (6). In our study, all patients were early onset, and a broad range of complications including RTI and autoimmune manifestations were reported. Moreover, gastrointestinal complications including chronic diarrhea were seen in all patents.

Treatment of the autoimmunity, chronic diarrhea and associated colitis is challenging in patients with antibody deficiency $(17,18)$. Uzzan et al. (19) reported that Ig supplementation does not significantly effect on the course of non-infectious gastrointestinal disease in CVID patients. Currently available treatments including steroids and cyclosporine have resulted in remission only in a subset of patients, then large doses of steroids are often necessary to control active disease. Patients with autoimmune entheropathy commonly do not respond to conventional treatment or other non-specific immune suppression therapies, however autoimmune entheropathy has been reported with partial response to immunosuppressive drugs such as cyclosporine, azathioprine and 6-mercaptopurine (20). Tacrolimus has been used as a treatment option for enteropathies, especially in autoimmune patients. Its mechanism of action is similar to cyclosporine. Both drugs block the gene activation for cytokine production by inhibiting the antigenic response of helper $T$ lymphocytes (21). Bousvaros et al. (16) in 1996 used tacrolimus for the first time as an alternative therapy for autoimmune enteropathy, and concluded that it can be efficacious when other immunosuppressive regimens fail (16). Mycophenolate mofetil has been also proposed as an alternative therapeutic option after the successful induction of remission in an infant with autoimmune enteropathy (22).

In the present report, we used sirolimus for clinical management of entropathy in patients with LRBA deficiency. We showed that disease symptoms such as chronic diarrhea and weight loss were successfully controlled after administration of sirolimus. Massey et al. (23) reported effectiveness of sirolimus in treatment of refractory Crohn's disease in an adult patient. Mutalib et al. (24) showed that sirolimus, by inducing both clinical remission and mucosal healing, is effective in children with severe IBD refractory to conventional therapies. In another study, Araki et al. (25) found that treatment of a severe refractory colonic and perianal Chrone's disease with sirolimus may result in a marked improvement in symptoms of enteropathy. Yong et al. (26) also reported the impact of sirolimus in children with IPEX and IPEX-like entheropathy. Although satisfying results of ad-

Table 1 - Immunologic characteristics of patients with LRBA deficiency underwent sirolimus therapy.

\begin{tabular}{|c|c|c|c|c|c|}
\hline Parameters & Patient 1 & Patient 2 & Patient 3 & Patient 4 & Normal Range \\
\hline $\operatorname{IgG}(\mathrm{mg} / \mathrm{dL})$ & 0 & 765 & 111 & 360 & $656-1351$ \\
\hline $\operatorname{IgM}(\mathrm{mg} / \mathrm{dL})$ & 0 & 69 & 28 & 44 & $34-255$ \\
\hline $\operatorname{IgA}(\mathrm{mg} / \mathrm{dL})$ & 4 & 5 & 24 & 0 & $86-320$ \\
\hline $\operatorname{IgE}(\mathrm{IU} / \mathrm{Ml})$ & 0 & 0.3 & 3 & - & Up to 46 \\
\hline White blood cell count $(\mathrm{cell} / \mu \mathrm{L})$ & 8210 & 8730 & 14700 & 14400 & $4000-11000$ \\
\hline Lymphocytes $($ cell/ $/ \mu \mathrm{L})$ & 2320 & 2095 & 7497 & 2016 & $1000-2800$ \\
\hline $\mathrm{CD}^{+}(\mathrm{cell} / \mu \mathrm{L})$ & 1717 & 1739 & 4273 & 1492 & $700-2100$ \\
\hline $\mathrm{CD}^{+}(\mathrm{cell} / \mu \mathrm{L})$ & 116 & 649 & 1724 & 363 & $300-1400$ \\
\hline $\mathrm{CD}^{+}(\mathrm{cell} / \mu \mathrm{L})$ & 1554 & 670 & 2624 & 1109 & $200-900$ \\
\hline $\mathrm{CD}^{+} / \mathrm{CD}^{+}{ }^{+}$ratio & 0.07 & 0.968 & 0.66 & 0.32 & $1-3$ \\
\hline CD16-56+ (cell/ $\mu \mathrm{L})$ & 819 & 98 & - & - & $90-600$ \\
\hline $\mathrm{CD} 9^{+}(\mathrm{cell} / \mu \mathrm{L})$ & 93 & 147 & 675 & 121 & $100-500$ \\
\hline
\end{tabular}


ministration of sirolimus have been documented in patients with autoimmune enteropathy prior to our study $(26,27)$, there was no evidence of using sirolimus in treatment of enteropathy in LRBA deficient patients. In the current study, for the first time, four LRBA deficient patients unresponsive to non-specific immune-suppressive agents underwent sirolimus therapy. Following administration of sirolimus, the frequency of diarrhea decreased and the patients' weight gradually normalized. Therefore, sirolimus with its potential efficacy and immunomodulatory properties may be recommended for the treatment of severe enteropathy in LRBA deficiency. Further studies should be designed to provide evidence for the effectiveness of sirolimus administration in management of diarrhea in immunodeficient patients by providing detailed pathological and microbiological evidences after treatment.

\section{References}

1. Lopez-Herrera G, Tampella G, Pan-Hammarstrom Q, Herholz P, Trujillo-Vargas CM, Phadwal K, et al. Deleterious mutations in LRBA are associated with a syndrome of immune deficiency and autoimmunity. American journal of human genetics. 2012;90(6):986-1001. PubMed PMID: 22608502. Pubmed Central PMCID: 3370280.

2. Alkhairy OK, Abolhassani H, Rezaei N, Fang M, Andersen KK, Chavoshzadeh Z, et al. Spectrum of Phenotypes Associated with Mutations in LRBA. Journal of clinical immunology. 2016;36(1):33-45. PubMed PMID: 26707784.

3. Azizi G, Rezaei N, Kiaee F, Tavakolinia N, Yazdani R, Mirshafiey A, et al. T-Cell Abnormalities in Common Variable Immunodeficiency. Journal of investigational allergology \& clinical immunology. 2016;26(4):233-43. PubMed PMID: 27374799.

4. Yazdani R, Abolhassani H, Rezaei N, Azizi G, Hammarstrom L, Aghamohammadi A. Evaluation of Known Defective Signaling-Associated Molecules in Patients Who Primarily Diagnosed as Common Variable Immunodeficiency. International reviews of immunology. 2016;35(1):7-24. PubMed PMID: 26959802.

5. Burns SO, Zenner HL, Plagnol V, Curtis J, Mok K, Eisenhut M, et al. LRBA gene deletion in a patient presenting with autoimmunity without hypogammaglobulinemia. The Journal of allergy and clinical immunology. 2012;130(6):1428-32. PubMed PMID: 22981790. Pubmed Central PMCID: 3930010.

6. Alangari A, Alsultan A, Adly N, Massaad MJ, Kiani IS, Aljebreen A, et al. LPS-responsive beige-like anchor (LRBA) gene mutation in a family with inflammatory bowel disease and combined immunodeficiency. The Journal of allergy and clinical immunology. 2012;130(2):481-8 e2. PubMed PMID: 22721650. Pubmed Central PMCID: 3582381.

7. Azizi G, Abolhassani H, Asgardoon MH, Alinia T, Yazdani R, Mohammadi J, et al. Autoimmunity in common variable immunodeficiency: epidemiology, pathophysiology and management. Expert review of clinical immunology. 2016Sep16:1-15. PubMed PMID: 27636680.

8. Azizi G, Abolhassani H, Asgardoon MH, Shaghaghi S, Negahdari B, Mohammadi J, et al. Managing patients with side effects and adverse events to immunoglobulin therapy. Expert Rev Clin Pharmacol. 2016;9(1):91-102. PubMed PMID: 26496172.
9. Marefat H, Abolhassani H, Ghareje Daghi M, Azizi G, Aghamohammadi A. Early onset steroid induced posterior subcapsular cataract in a patient with common variable immunodeficiency: case reports and review of literature. European annals of allergy and clinical immunology. 2016;48(5):197-201. PubMed PMID: 27608477.

10. Serwas NK, Kansu A, Santos-Valente E, Kuloglu Z, Demir A, Yaman A, et al. Atypical manifestation of LRBA deficiency with predominant IBD-like phenotype. Inflamm Bowel Dis. 2015;21(1):40-7. PubMed PMID: 25479458.

11. Azizi G, Ahmadi M, Abolhassani H, Yazdani R, Mohammadi H, Mirshafiey A, et al. Autoimmunity in Primary Antibody Deficiencies. International archives of allergy and immunology. 2016;171(3-4):180-93. PubMed PMID: 28013299.

12. Azizi G, Abolhassani H, Rezaei N, Aghamohammadi A, Asgardoon MH, Rahnavard J, et al. The Use of Immunoglobulin Therapy in Primary Immunodeficiency Diseases. Endocrine, metabolic \& immune disorders drug targets. 2016Jul24. PubMed PMID: 27456825 .

13. Teahon K, Webster AD, Price AB, Weston J, Bjarnason I. Studies on the enteropathy associated with primary hypogammaglobulinaemia. Gut. 1994;35(9):1244-9. PubMed PMID: 7959231. Pubmed Central PMCID: PMC1375701.

14. Cunningham-Rundles C. How I treat common variable immune deficiency. Blood. 2010;116(1):7-15. PubMed PMID: 20332369. Pubmed Central PMCID: 2904582. Epub 2010/03/25. eng.

15. Charbonnier LM, Janssen E, Chou J, Ohsumi TK, Keles S, Hsu JT, et al. Regulatory T-cell deficiency and immune dysregulation, polyendocrinopathy, enteropathy, X-linked-like disorder caused by loss-of-function mutations in LRBA. The Journal of allergy and clinical immunology. 2015xw;135(1):217-27. PubMed PMID: 25468195. Pubmed Central PMCID: 4289093.

16. Bousvaros A, Leichtner AM, Book L, Shigeoka A, Bilodeau J, Semeao E, et al. Treatment of pediatric autoimmune enteropathy with tacrolimus (FK506). Gastroenterology. 1996;111(1):237-43. PubMed PMID: 8698205. Epub 1996/07/01. eng.

17. Azizi G, Ziaee V, Tavakol M, Alinia T, Yazdai R, Mohammadi $\mathrm{H}$, et al. Approach to the Management of Autoimmunity in Primary Immunodeficiency. Scandinavian journal of immunology. 2017;85(1):13-29. PubMed PMID: 27862144.

18. Azizi G, Mirshafiey A. Imatinib mesylate: an innovation in treatment of autoimmune diseases. Recent patents on inflammation \& allergy drug discovery. 2013;7(3):259-67. PubMed PMID: 23947692.

19. Uzzan M, Ko HM, Mehandru S, Cunningham-Rundles C. Gastrointestinal Disorders Associated with Common Variable Immune Deficiency (CVID) and Chronic Granulomatous Disease (CGD). Curr Gastroenterol Rep. 2016;18(4):17. PubMed PMID: 26951230. Pubmed Central PMCID: PMC4837890.

20. Gentile NM, Murray JA, Pardi DS. Autoimmune enteropathy: a review and update of clinical management. Curr Gastroenterol Rep. 2012;14(5):380-5. PubMed PMID: 22810979. Pubmed Central PMCID: 3912565. Epub 2012/07/20. eng.

21. Vanderhoof JA, Young RJ. Autoimmune enteropathy in a child: response to infliximab therapy. J Pediatr Gastroenterol Nutr. 2002;34(3):312-6. PubMed PMID: 11964960. Epub 2002/04/20. eng.

22. Baud O, Goulet O, Canioni D, Le Deist F, Radford I, Rieu $\mathrm{D}$, et al. Treatment of the immune dysregulation, poly- 
endocrinopathy, enteropathy, X-linked syndrome (IPEX) by allogeneic bone marrow transplantation. N Engl J Med. 2001;344(23):1758-62. PubMed PMID: 11396442. Epub 2001/06/09. eng.

23. Massey DC, Bredin F, Parkes M. Use of sirolimus (rapamycin) to treat refractory Crohn's disease. Gut. 2008;57(9):1294-6. PubMed PMID: 18719139.

24. Mutalib M, Borrelli O, Blackstock S, Kiparissi F, Elawad M, Shah $\mathrm{N}$, et al. The use of sirolimus (rapamycin) in the management of refractory inflammatory bowel disease in children. Journal of Crohn's and Colitis. 2014;8(12):1730-4.
25. Araki K, Ellebedy AH, Ahmed R. TOR in the immune system. Curr Opin Cell Biol. 2011;23(6):707-15. PubMed PMID: 21925855. Pubmed Central PMCID: PMC3241972.

26. Yong PL, Russo P, Sullivan KE. Use of sirolimus in IPEX and IPEX-like children. Journal of clinical immunology. 2008;28(5):5817. PubMed PMID: 18481161. Epub 2008/05/16. eng.

27. Bindl L, Torgerson T, Perroni L, Youssef N, Ochs HD, Goulet $\mathrm{O}$, et al. Successful use of the new immune-suppressor sirolimus in IPEX (immune dysregulation, polyendocrinopathy, enteropathy, X-linked syndrome). J Pediatr. 2005;147(2):256-9. PubMed PMID: 16126062. Epub 2005/08/30. eng. 